\title{
The Analysis of PPG Morphology: Investigating the Effects of Aging on Arterial Compliance
}

\author{
Q. Yousef ${ }^{1}$, M. B. I. Reaz ${ }^{2}$, M. A. M. Ali ${ }^{2}$ \\ ${ }^{1}$ College of computer science and engineering Taibah University, Madinah-Saudi Arabia \\ ${ }^{2}$ Department of Electrical, Electronic and Systems Engineering, \\ University Kebangsaan Malaysia, 43600 UKM, Bangi, Selangor, Malaysia
}

\begin{abstract}
This study presents the variations of photoplethysmogram (PPG) morphology with age. PPG measurement is done noninvasively at the index finger on both right and left hands for a sample of erectile dysfunction (ED) subjects. Some parameters are derived from the analysis of PPG contour showed in association with age. The age is found to be an important factor that affects the contour of PPG signals which accelerates the disappearance of PPG's dicrotic notch and PPG's inflection point as well. Arterial compliance is found to be degraded with age due to the fall of arterial elasticity. This study approaches the establishment of usefulness of PPG's contour analysis as an investigator to the changes in the elastic properties of the vascular system, and as a detector of early sub-clinical atherosclerosis.
\end{abstract}

Keywords: Carotid intima-media thickness, contour analysis, erectile dysfunction, photoplethysmogram, reflection index, stiffness index

\section{INTRODUCTION}

E RECTILE DYSFUNCTION (ED) is a sign of cardiovascular disease which can be a sign of increased risk [1]. The risk of cardiovascular diseases may damage the endothelium cells which may cause atherosclerosis (stiffening of arteries). Arterial stiffness is not only a marker of cardiovascular dysfunction but also an independent risk factor for cardiovascular disease [2]-[6]. Pulse pressure, a surrogate marker of increased arterial stiffness, is a powerful predictor of cardiovascular events [7] [9] [25]. As large arteries stiffen with age or disease processes, the amplitude of the reflected wave increases [3].

Arterial stiffness can be measured noninvasively by the use of the PPG technique, which reflects the changes in blood volume with each heart beat. Perhaps the most exciting application of PPG waveform analysis is the possibility of providing a rapid biophysical measure of diseases or aging process [10]. PPG provides an estimation of coetaneous blood flow by measuring the dynamic attenuation of infrared light by the blood volume present in tissue [11]. Analysis of the PPG's contour in terms of crest time revealed age dependence and clinically significant variations between health and disease, including arteriosclerosis, hypertension, and various dermatoses [12]. Arterial pulsations are the most significant portion of PPG [13]. PPG's oscillating component provides a pulsatile wave, whose contour may include content descriptive of vascular health [11], [14]-[15]. The PPG waveform comprises a pulsatile (AC) physiological waveform attributed to cardiac synchronous changes in the blood volume with each heart beat [16]. PPG measurements were done on the index finger (both right and left hands) since finger PPG is a commonly used technique in medicine and since it is easier for recording [17]-[18].

Derivations of quantitative measures characterizing pulse shape have proven useful in analyzing the PPG signal [11]. The peripheral pulse is often used in the assessment of health and disease [17]. Fig.1 shows the characteristics of PPG, the peaks position and timing are utilized in calculating PPG's indices. Reflection index (RI) is derived as a ratio of pulse inflection peak amplitude (second peak) over the pulse max amplitude. RI can provide a window to vascular age and arterial compliance. RI mainly depends on the detection of PPG second peak which tends to be less pronounced with aging. The systole, diastole, and dicrotic notch points over PPG contour were located and calculated by an optimized algorithm developed in Matlab.

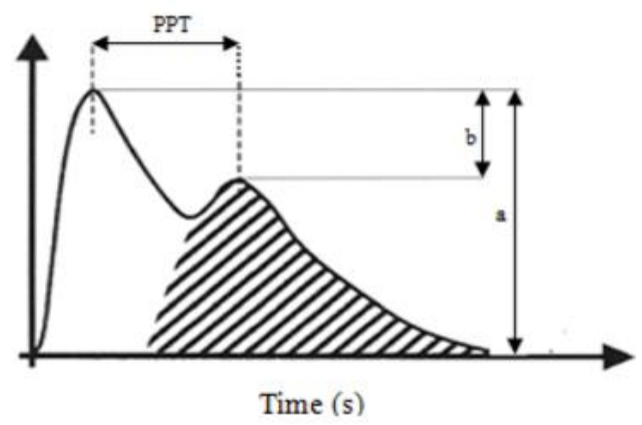

Fig.1. The characteristics of PPG

\section{MATERIALS \& METHODS}

\section{A. Data acquisition for PPG analysis}

PPG pulse measurements are collected simultaneously on the index finger (both left and right) to study and analyze arterial conditions. All patients have an established ED which differs in the amount of its degree from one patient to another. After subjects rest for 5 minutes (to ensure cardiovascular stability), PPG recordings are carried out for the duration of 90 seconds. During the measurements, subjects are kept quiet, and breathe normally while resting in a supine position. PPG measurements are performed in hospital conditions at room temperature $\left(24 \pm 1^{\circ} \mathrm{C}\right)$. 


\section{B. Hardware setup for signal processing}

A special National Instruments with Data Acquisition board (NI cDAQ-9172) is used to digitize the signals locally and transmit the digital data to a personal computer with sampling rate of $5500 \mathrm{~Hz}$. The recorded signals are analyzed off-line using customized algorithm developed in MATLAB (The MathWorks, Inc). PPG signals are down-sampled (275 $\mathrm{Hz}$ ), de-trended for removing outliers, drifts, offset, and any movement artifacts. Next, PPG signals are band-pass filtered $(0.6-15 \mathrm{~Hz})$ to remove the effects of respiratory rhythm and higher frequency disturbances [19]. These filters do not introduce phase delays or distortion to the waveforms [20]. Utilizing PPG derivatives, those points of interest can be located and determined [20]-[23]. Fig.2 shows PPG signal, PPG $1^{\text {st }}$ derivative, PPG $2^{\text {nd }}$ derivative, and the process of peak detection.

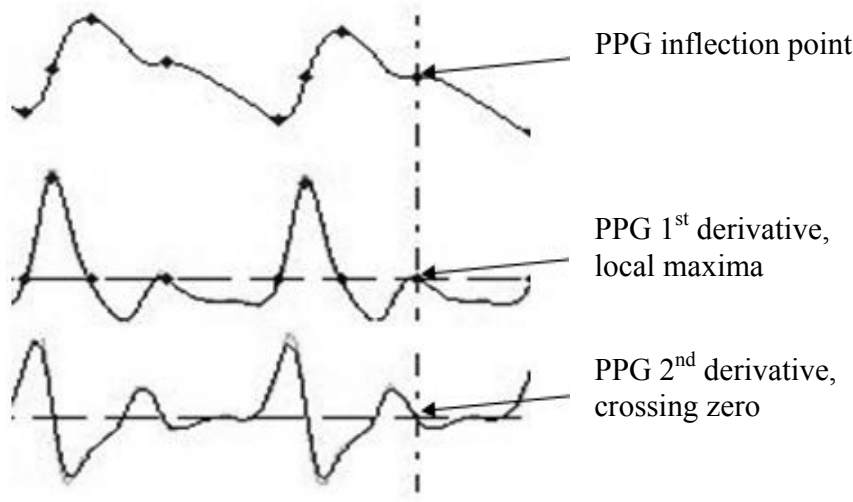

Fig.2. PPG signal, PPG $1^{\text {st }}$ derivative, $P P G 2^{\text {nd }}$ derivative, and the process of peak detection

RI has shown to be a noninvasive indicator for vascular assessment. RI can be calculated based on (1):

$$
R I=\frac{b}{a} * 100 \%
$$

where $b$ or DM is the amplitude of inflection peak (second peak), and, $a$ or PM is the maximum amplitude of the pulse.

Basically, PPG signals which experience a clearly seen second peak seem to be visualized in healthy young subjects, while they are not pronounced clearly in old subjects; however, they tend to be less pronounced or unseen in most cases. These observations are not applicable for all cases, since we still have the opposite situation in some other cases for each observation. However, RI remains a good measure for arterial compliance. In addition, augmentation index (AI) was derived in terms of pulse maximum peak and PPG second peak as well. AI can be used as a measure of arterial stiffness.

$$
A I=\frac{P s-P d}{P i-P d}
$$

Where Pi is peak systolic pressure, Ps is an inflection point that indicates the beginning up-stroke of the reflected pressure wave, and $\mathrm{Pd}$ is minimum diastolic pressure.
Both RI and AI depend completely on PM and DM parameters. PM appears to be correlated negatively with age, which reflects arterial compliance and can provide a window to arterial elasticity. Healthy artery shall be low in resistance which in turn increases the compliance, while aging artery is high in resistance which decreases the compliance. Thereby, PM can be used as a measure of arterial compliance since it is going to be reduced with age.

The demonstration of correlations between parameters of peripheral pulse wave analysis and pulse wave velocity (PWV) measured both invasively and noninvasively supports the revival of PPG as a simple and inexpensive technique for the assessment of vascular health [11], [23], [24]. Stiffness index (SI) is a measure of larger artery stiffness, can be used as a surrogate measure of PWV, correlates negatively with age. SI is calculated using (2) in terms of the subject's height divided by pulse transit time (PTT or $\Delta \mathrm{T}$ ). The peripheral pulse wave at the finger characteristically exhibits a systolic peak resulting from the direct pressure wave traveling from the left ventricle to the digit, and a diastolic peak or inflection resulting from reflections of the pressure wave by arteries of the lower body back to the finger [11]. During age advance, diastolic peak tends to be closer to systolic peak which, in turn, reduces PTT and increased SI:

$$
\mathrm{SI}=\frac{\mathrm{H}}{\Delta \mathrm{T}}
$$

where $\mathrm{H}$ is the subject's height.

This paper aims to investigate the effects of aging on arterial elastic properties and on the variations of PPG contour as well. This paper reports the usefulness of PPG contour analysis in investigating and studying cardiovascular activities by relating the variations of its morphology to any changes in cardiovascular system. However, observing and recording changes in PPG waveform might result in certain changes or abnormality in one or more cardiovascular parameters. The effect of age in PPG's contour, data preprocessing and the differences between the left and right hand index finger were also examined.

\section{Erectile dysfunction subjects}

PPG measurements were collected from the right and left index fingers of 65 participants with ages ranging from 30 to 78 years and median age 56 years (all men). Eleven subjects were excluded due to missing information (age or height), unacceptable pulse volume data and/or noisy signal at either right or left index finger PPG. A written consent was taken from each participant. The data were recorded from a longitudinal study initially undertaken for the assessment of endothelium dysfunction in subjects with erectile dysfunction.

Table 1 shows the medical data characteristics for each age group. Carotid intima-media thickness (CIMT) test, body mass index (BMI), systolic pressure (SP), diastolic pressure (DP), pulse pressure (PP), and main arterial pressure (MAP) were taken in the same stage of data collection. Subjects are from three different races in Malaysia (Malay, Chinese, and Indian). 
Table 1. Medical data characteristics for each age group (median \pm SD)

\begin{tabular}{|c|c|c|c|c|c|c|}
\hline $\begin{array}{l}\text { Age } \\
\text { (yrs) }\end{array}$ & CIMT & BMI & SP & DP & PP & MAP \\
\hline $30-40$ & $.467 \pm 0.11$ & $23.4 \pm 0.35$ & $130 \pm 10$ & $80 \pm 10$ & $50 \pm 0$ & $96 \pm 10$ \\
\hline $41-50$ & $.63 \pm 0.22$ & $26.3 \pm 4.7$ & $135.5 \pm 10.5$ & $582 \pm 6$ & $53.5 \pm 6.7$ & $7100 \pm 7$ \\
\hline $51-60$ & $.92 \pm 0.37$ & $26.8 \pm 5.4$ & $138 \pm 11$ & $81.6 \pm 6.2$ & $256 \pm 12$ & $100 \pm 6$ \\
\hline$>60$ & $.90 \pm 0.38$ & $26.7 \pm 3.5$ & $141 \pm 9$ & $85 \pm 8.2$ & $56 \pm 7.2$ & $103 \pm 8$ \\
\hline \multicolumn{7}{|c|}{$\begin{array}{l}\text { CIMT, carotid intima--media thickness }(\mathrm{mm}) \text {; BMI, body mas } \\
\text { index }\left(\mathrm{kg} / \mathrm{m}^{2}\right) \text {; } \mathrm{SP} \text {, systole blood pressure }(\mathrm{mmHg}) \text {; DP, diastol } \\
\text { blood pressure }(\mathrm{mmHg}) \text {; } \mathrm{PP} \text {, pulse pressure }(\mathrm{mmHg}) \text {; MAP, mair } \\
\text { arterial pressure }(\mathrm{mmHg}) \text {. }\end{array}$} \\
\hline
\end{tabular}

Main arterial pressure (MAP) and pulse pressure (PP) are calculated based on equations 3 and 4, respectively:

$$
\begin{gathered}
M A P \cong \frac{1}{3} * S B P+\frac{2}{3} D B P \\
P P=S P-D P
\end{gathered}
$$

\section{Protocol}

The study was conducted in Urology Clinic in the National University of Malaysia Medical Centre (PPUKM). PPUKM is a teaching medical center with 750 beds. The medical center provides health services to most of the population around Kuala Lumpur as well as Selangor state. The study was approved by the PPUKM ethics community review. Each patient was informed about the details of the study and their written consent was taken before the recordings were made.

The baseline examination included medical history taking, physical examination, laboratory testing, and assessment of cardiovascular disease status, and blood pressure measurement have been investigated and recorded. Subjects were to meet some inclusion criteria (hypertension, diabetes mellitus, dyslipidemia, obesity, smoking, and significant family history) and no established cardiovascular disease or risks at all. This variety in inclusion criteria was just to form control (no risk) and case (risk) groups. However, there were some exclusion criteria as well (established cardiovascular disease, liver cirrhosis, renal failure, thyroid disease, and spinal cord or finger injuries, or having Raynauld's).

\section{E. Statistical analysis}

The data are quantitative variables summarized by means and standard deviations. In analyzing periodic signals, the mean and median are the most important parameters [8]. Therefore, median value is used to represent the extracted indices. The Pearson correlation is used to characterize the relationship between continuous variables (SPSS Release 11.5.0). The box plot and scatter plot are performed using Matlab (R2008a). Table 2 demonstrates the correlation between some PPG indices for both right and left hand PPG.

The result reveals a strong correlation between amplitude PPG indices, while it also reveals an exact result for timing PPG indices. By observing Table 2, RI, AI, PM, and DM seem to be highly correlated (their calculation are based on volume changes), while SI, PT, DiT, and $\Delta \mathrm{T}$ are exactly equal (their calculations are based on time changes).
Table 2. Paired sample correlations

\begin{tabular}{lll}
\hline Parameter & Correlation & Significant $(P)$ \\
\hline RIR and RIL & 0.703 & 0.000 \\
SIR and SIL & 1.000 & 0.000 \\
AIR and AIL & 0.892 & 0.000 \\
PMR and PML & 0.565 & 0.000 \\
DMR and DML & 0.753 & 0.000 \\
PTR and PTL & 1.000 & 0.000 \\
DiTR and DiTL & 0.999 & 0.000 \\
$\Delta$ TR and $\Delta$ TL & 1.000 & 0.000 \\
\hline
\end{tabular}

\section{RESULTS AND DISCUSSION}

The investigated PPG parameters of contour analysis are derived from measures of locating points of interest on the contour of PPG and they calculate those indices in terms of timing and amplitude changes. Changes in time (pulsation time) reflect cardiovascular rhythm and the power of left ventricle. Consequently, changes in amplitude (pulsation amplitude) reflect arterial wall stiffness. Our results indicate that these parameters significantly correlate with one another. However, up to our knowledge, this is the first time that the correlation between PPG indices and age in erectile dysfunction subjects has been demonstrated and correlated with CIMT test. It is noteworthy that PPG time indices are equal; such observation shall be utilized to distinguish between normal and abnormal signals.

PPG parameters were found to be affected by age even when the relationship was not linear. The contour of PPG pulse changed clearly with age. SI was noticed to be increased with age due to the observation that $\Delta \mathrm{T}$ tends to be decreased with age. The result supports the findings of Anne et al., since there was no age-based evidence to affect PPG indices in the right and left hand fingers (our study was on the index finger while the Anne et al. study was on the middle finger).

The analysis of the PPG amplitude (AC pulsations) is very important. The changes in blood volume (measured by PPG) provide abundant information about arterial compliance and arterial elastic properties. As arteries lose their elastic properties with age, PPG volume tends to be declined, reflecting the effect of arteriosclerosis on the propagation of blood stream. Indices utilizing the variations on PPG amplitude can provide a window to detect early arteriosclerosis in individuals experiencing one or more CVDs. The time for each pulse period of PPG signal (PT) can be calculated as the difference in time between each two sequential pulses. The relation between PT and age is illustrated in Fig.3. The result reveals the trend of PT to be increased with age. Such observations relate PPG's contour to be altered with age, which reflects the changes of arterial characteristics due to aging.

The maximum amplitude of PPG's single pulse (PM) contributes to the compliance of arteries which tends to be declined with age (Fig.4). This phenomenon represents the response of arterial wall to blood pressure. In healthy (no or less arteriosclerosis) arteries, the resistance of artery wall to that pressure is low and the capacitance is high. If the process of arteriosclerosis is started, then the resistance will be higher and the capacitance will be declined, thereby affecting the amplitude of PPG pulsations to be reduced. 


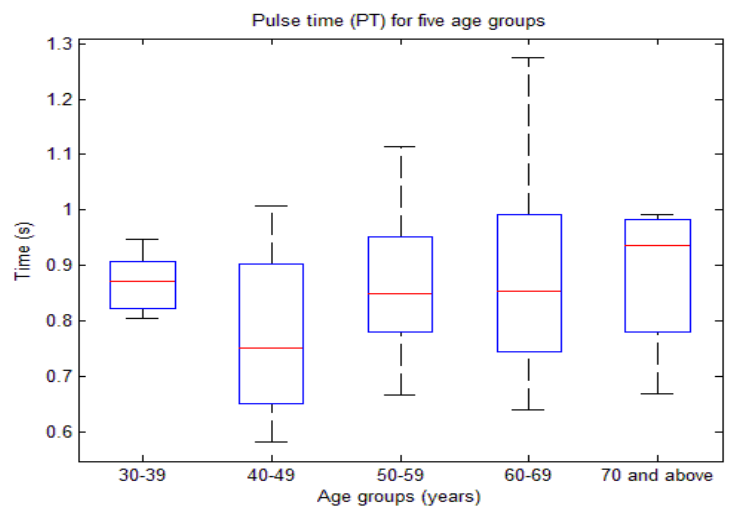

Fig.3. PPG pulse timing for five age groups

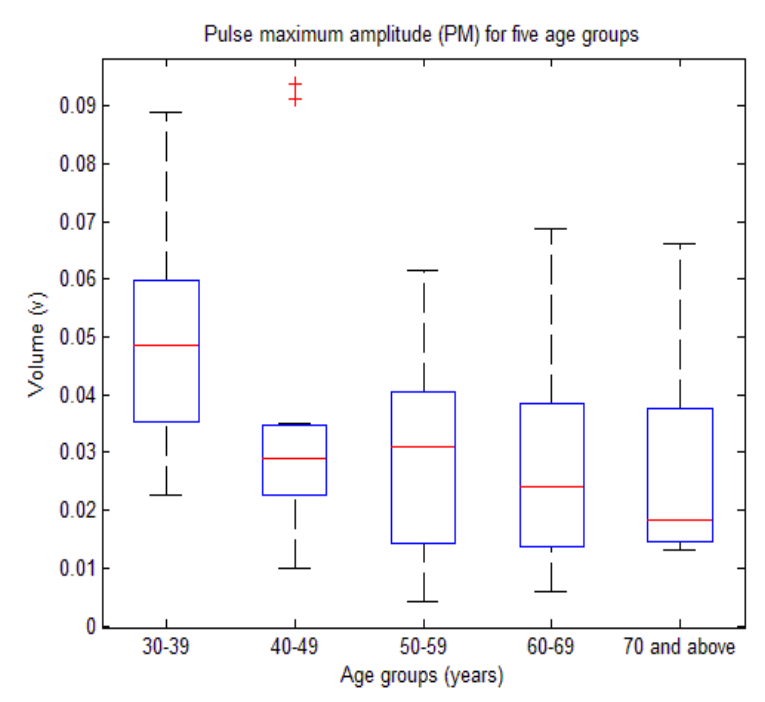

Fig. 4. Box plot for PM of five age groups

$\mathrm{RI}$ is derived as a measure of PPG amplitude changes based on the detection of systole and inflection point. Fig.5 represents Box plot for RI based on five age groups. It is clearly seen that age is affecting the variations of the RI parameter. By observing the changes among each age group, RI seems to be increasing from one age group to the next, which in turn strengthens the effects of aging on PPG contour variations.

$\mathrm{RI}$ is subjected to be increased with age due to the reduction in PM amplitude and to the increment of the DM amplitude due to the augmentation of diastole in systole part with age. This phenomenon is the reason behind the inflection point being close to systole point. Observing the variations of PPG signal in different age groups strengthens these findings. With age, PPG contour becomes more rounded which is observed by less pronounced dicrotic notch and inflection point.

PPG augmentation index has proven to be a noninvasive indicator for vascular assessments [26]. Fig.6 shows that AI differs slightly from the right to left hand. However, the correlation between AIR and AIL is $0.892, P<0.000$. The findings for RI and $\mathrm{AI}$ are in agreement with the findings of Rubins et al. [22] (AI and RI calculated from 2 PPG sites (middle finger and ear)).

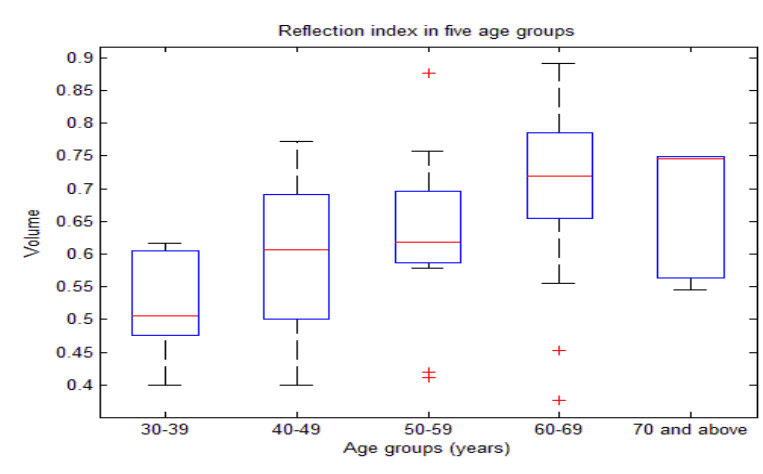

Fig.5. RI among five age groups

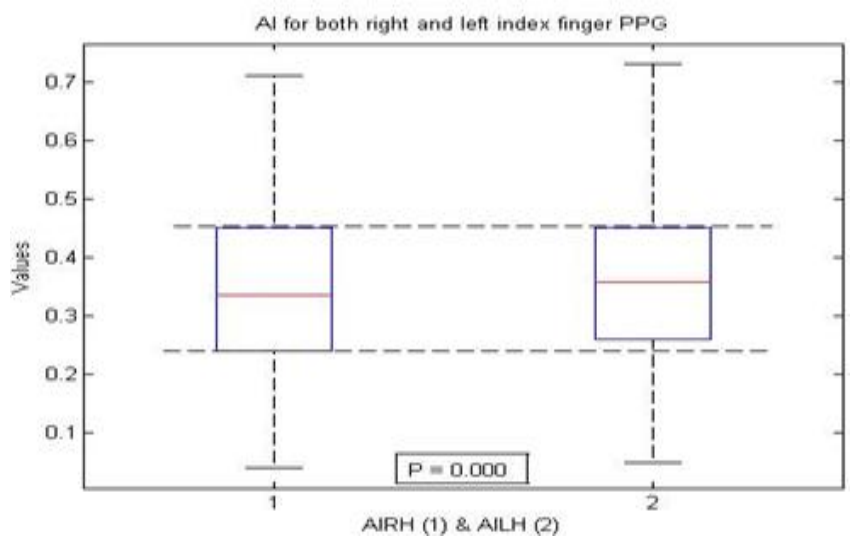

Fig.6. Box plot for AI right hand and AI left hand

With age, the time to reach inflection point (DiT) tends to be increased. Statistics showed a good correlation between age and DiT since age correlates with DiTR $(0.295, P=$ $0.03)$ and with DiTL $(0.301, P=0.03)$. A CIMT test, which is the gold standard test for arteriosclerosis, was found to be associated with SI and $\Delta \mathrm{T}$. The observation is that an increment in CIMT value will result in a reduction of $\Delta \mathrm{T}$ which in turn affects SI to be increased as CIMT increases. Arteriosclerosis is affecting arterial elasticity, thereby increasing arterial stiffness with age. PPG measurements were made using both the right and left hand index fingers, hypothesizing that there would be little or no difference between the two sites of measurements. However, looking for the association between age and PPG is a great idea, but one should realize that such a relation is not linear. Researchers thought that, in looking for the age effects on PPG, PPG should better be considered as a whole pulse, not as a single region or single parameter. Investigation and study of age-related changes to the peripheral pulse contour using a much larger and diverse population is necessary for the establishment of important aging indices [11].

\section{CONCLUSIONS}

PPG contour analysis provides a window to cardiovascular activities. This study proposed assessing PPG contour variations based on PPG data recording from right and left index fingers. The study concluded that PPG's timing indices were equal in both right and left hand, this might 
indicate the stability of pulse time in both left and right sides. In addition, it concluded that PPG's amplitude indices differ slightly for right and left hand but still have a strong correlation with each other. A beat-to-beat change of the PPG amplitude is often the first clue that the patient has developed an irregular heart rhythm. The amplitude variations shall be given more efforts and attention in order to ride the challenge in developing some means of pulse contour, which can be used to assist in health and disease and to be applied into clinical settings. Age is an important factor in arterial stiffness, since, as we age, arteriosclerosis increases. In older subjects, the arteries are less distensible, leading to high SI and high RI, thereby resulting in a more rounded PPG with a lack of dicrotic notch which in turn decreases arterial compliance. Analysis of PPG contour might have important clinical implications which in turn strengthen the suggestion that PPG offers a fruitful avenue for new technologic developments in noninvasive circulatory monitoring.

\section{REFERENCES}

[1] WebMD. (2012). Atherosclerosis and erectile dysfunction. www.webmd.com/erectile-dysfunction/ atherosclerosis-and-erectile-dysfunction.

[2] Arnett, D.K., Evans, G.W., Riley, W.A. (1994). Arterial stiffness: A new cardiovascular risk factor? American Journal of Epidemiology, 140 (8), 669-682.

[3] Klocke, R., Cockcroft, J.R., Taylor, G.J., Hall, I.R., Blake, D.R. (2003). Arterial stiffness and central blood pressure, as determined by pulse wave analysis, in rheumatoid arthritis. Annals of the Rheumatic Diseases, 62 (5), 414-418.

[4] Reaz, M.B.I., Muhammad, I.I., Rosminazuin, A.R. (2006). Neural network based ECG signal analysis for heart rate detection. WSEAS Transactions on Signal Processing, 2 (6), 889-895.

[5] Reaz, M.B.I., Muhammad, I.I, Rosminazuin, A.R. (2006). An approach to detect QRS complex using backpropagation neural network. In Proceedings of the $7^{\text {th }}$ WSEAS International Conference on Neural Networks, 12-14 June 2006. Wisconsin, US: WSEAS, 28-33.

[6] Reaz, M.B.I., Wei, L.S., (2004). Adaptive linear neural network filter for fetal ECG extraction. In Proceedings of International Conference on Intelligent Sensing and Information Processing (ICISIP 2004), 5-7 January 2004. IEEE, 321-324.

[7] Franklin, S.S., Khan, S.A., Wong, N.D., Larson, M.G., Levy, D. (1999). Is pulse pressure useful in predicting risk for coronary heart disease? The Framingham heart study. Circulation, 100 (4), 354-360.

[8] Hussain, M.S., Mamun, M.D. (2012). Effectiveness of the wavelet transform on the surface EMG to understand the muscle fatigue during walk. Measurement Science Review, 12 (1), 28-33.

[9] Ibrahimy, M.I., Reaz, M.B.I., Ali, M.A.M., Khoon, T.H., Ismail, A.F. (2006). Hardware realization of an efficient fetal QRS complex detection algorithm. WSEAS Transactions on Circuits and Systems, 5 (4), 575-581.
[10] Huotari, M., Yliaska, N., Lantto, V., Määttä, K., Kostamovaara, J. (2009). Aortic and arterial stiffness determination by photoplethysmographic technique. Procedia Chemistry, 1 (1), 1243-1246.

[11] Brumfield, A.M., Andrew, M.E. (2005). Digital pulse contour analysis: Investigating age-dependent indices of arterial compliance. Physiological Measurement, 26 (5), 599-608.

[12] Gavish, B. (1987). Photoplethysmographic characterization of the vascular wall by a new parameter- minimum rise-time: Age dependence on health. Microcirc Endothelium Lymphatics, 3 (3-4), 281-296.

[13] Reisner, A., Shaltis, P.A., McCombie, D., Asada, H.H. (2008). Utility of the photoplethysmogram in circulatory monitoring. Anesthesiology, 108 (5), 950958.

[14] Challoner, J. (1979). Photoelectric Plethysmography for Estimating Cutaneous Blood Flow Non-Invasive Physiological Measurements. London: Academic Press, 127-151.

[15] Millasseau, S.C., Kelly, R.P., Ritter, J.M., Chowienczyk, P.J. (2002). Determination of agerelated increases in large artery stiffness by digital pulse contour analysis. Clinical Science, 103, 371-377.

[16] Mohamed, S., Mahamod, I., Zainol, R. (2004). Artificial neural network (ANN) approach to PPG signal classification. International Journal of Computing \& Information Sciences, 2 (1), 58-65.

[17] Allen, J., Frame, R., Murray, A. (2002). Microvascular blood flow and skin temperature changes in the fingers following a deep inspiratory gasp. Physiological Measurement, 23 (2), 365-373.

[18] Allen, J. (2007). Photoplethysmography and its application in clinical physiological measurement. Physiological Measurement, 28 (3), R1.

[19] Mohd-Yasin, F., Yap, M.T., Reaz, M.B.I. (2007). CMOS instrumentation amplifier with offset cancellation circuitry for biomedical application. WSEAS Transactions on Circuits and Systems, 6 (1), 171-174.

[20] Edmond, Z., Kalaivani, C., Mohd, A., Harwant, S. (2007). Analysis of the effect of ageing on rising edge characteristics of the photoplethysmogram using a modified Windkessel model. Cardiovascular Engineering, 7 (4), 172-181.

[21] Karamanoglu, M. (1997). A system for analysis of arterial blood pressure waveforms in humans. Computers and Biomedical Research, 30 (3), 244-55.

[22] Rubins, U., Grube, J., Kukulis, I. (2008). Photoplethysmography analysis of artery properties in patients with cardiovascular diseases. In $14^{\text {th }}$ NordicBaltic Conference on Biomedical Engineering and Medical Physics, 16-20 June 2008. Springer, 319-322.

[23] Qawqzeh, Y., Ali, M.A.M., Reaz, M.B.I., Maskon, O. (2010). Photoplethysmogram peaks analysis in patients presenting with erectile dysfunction. In Proceedings of the International Conference on Electrical Computer Technology (ICECT 2010), 7-10 May 2010, 165-168. 
[24] Loukogeorgakis, S., Dawson, R., Phillips, N., Martyn C.N., Greenwald, S.E. (2002). Validation of a device to measure arterial pulse wave velocity by a photoplethysmographic method. Physiological Measurement, 23 (3), 581-596.

[25] Hasan, M.A., Reaz, M.B.I., Ibrahimy, M.I., Hussain, M.S., Uddin, J. (2009). Detection and processing techniques of FECG signal for fetal monitoring. Biological Procedures Online, 11 (1), 263-295.
[26] Gonzalez, R., Delgado, A., Padilla, J., Trenor, B., Ferrero, J., Saiz J. (2008). Photoplethysmographic augmentation index as a non invasive indicator for vascular assessments. In 4th European Conference on the International Federation for Medical and Biological Engineering, 23-27 November 2008. Springer, 1167-1170.

Received May 19, 2012. Accepted November 21, 2012. 